\title{
Heart rate variability in sciatica patients referred to spine surgery: a case control study
}

\author{
Jarmo Södervall ${ }^{1,2}$, Jaro Karppinen ${ }^{2,3}$, Jukka Puolitaival ${ }^{2}$, Eero Kyllönen ${ }^{2}$, Antti M Kiviniemi ${ }^{1}$, Mikko P Tulppo \\ and Arto J Hautala ${ }^{*}$
}

\begin{abstract}
Background: A chronic pain condition may result in altered autonomic nervous system regulation in various patient populations. We evaluated whether autonomic regulation differs between sciatica patients referred to spine surgery and age-matched healthy controls analyzed with heart rate variability techniques (HRV).

Methods: HRV of patients $(n=201)$ and healthy controls $(n=138)$ were measured in standing conditions (5 min). High frequency (HF) power as an index of cardiac vagal modulation and the low-to-high-frequency (LF/HF) ratio and short-term fractal scaling exponent $a_{1}$ as indices of sympathovagal balance were analyzed. Pain intensity was assessed on a Visual Analog Scale (VAS) and perceived disability with Oswestry Disability Index.

Results: The Oswestry and VAS scores were higher in the patients than in the controls ( $p<0.0001$ for both). HF power was markedly lower for the patients compared to the controls $(p<0.0001)$. The LF/HF ratio and $a_{1}$ were higher in the patients than in the controls ( $p<0.01$ for both). After adjusting for sex, smoking, BMl, and leisure-time physical activity, HF power $(p=0.011)$ and $a_{1}(p=0.012)$ still differed between the groups. Among the patients, HF power was slightly associated with the duration of chronic pain $(r=-.232, p=0.003)$.

Conclusions: Sciatica patients referred to spine surgery had altered cardiac autonomic regulation expressed as decreased vagal activity and an increased sympathovagal balance toward sympathetic dominance when compared with age-matched healthy controls.
\end{abstract}

Keywords: Autonomic nervous system, Back pain, Sciatica, Sympathovagal balance, Vagal activity

\section{Background}

Low back disorders, including low back pain (LBP) and sciatica, are common health problems among adults and represent a significant burden on health care systems globally. A population-based survey showed that $19 \%$ of the subjects had chronic pain, and LBP was the main reason in almost half of these patients [1]. Sciatic pain is more severe and the outcome of this pain is worse than localized LBP [2]. Furthermore, it causes an increased risk of hospitalization [3], longer absences due to illness, and more disability than does nonspecific LBP $[4,5]$.

Various states of chronic pain are associated with altered autonomic nervous system function, assessed with the heart rate variability (HRV) indices, e.g. patients with

\footnotetext{
* Correspondence: arto.hautala@verve.fi

${ }^{1}$ Department of Exercise and Medical Physiology, Verve Research, Kasarmintie 13, P.O. Box 404, 90101 Oulu, Finland

Full list of author information is available at the end of the article
}

fibromyalgia have shown lower HRV [6] and blunted autonomic response to an orthostatic challenge [7]. Decreased HRV has also been reported in patients with complex regional pain syndrome [8] and in chronic neck-shoulder pain patients [9]. Likewise, signs of altered autonomic regulation in subjects with chronic LBP have been observed. An earlier study on chronic LBP showed that HRV was lower among patients with a moderate perceived disability (Oswestry 20-40\%) than among those with a minimal disability (Oswestry $<20 \%$ ). Interestingly, a significant association was found between HRV and perceived physical impairment, but not between HRV and subjective pain evaluated on a numerical rating scale [10].

Depressed HRV has been shown to be associated with an adverse prognosis in general populations as well as in different patient groups [11-16]. The function of the autonomic nervous system in sciatica patients referred
Ciomed Central

(c) 2013 Södervall et al.; licensee BioMed Central Ltd. This is an Open Access article distributed under the terms of the Creative Commons Attribution License (http://creativecommons.org/licenses/by/2.0), which permits unrestricted use, distribution, and reproduction in any medium, provided the original work is properly cited. 
to spine surgery is not well understood and, to our knowledge, has not been meticulously studied in relation to various spectral and non-linear dynamics of HRV. Therefore, we examined whether HRV differs between sciatica patients referred to spine surgery and agematched healthy controls. We hypothesized that HRV is altered in sciatica patients compared with healthy controls, and secondly, that HRV is associated with pain intensity and perceived disability in sciatica patients.

\section{Methods}

\section{Study population}

The study population consisted of voluntary consecutive patients $(n=201$, age $42 \pm 11$ years, 113 men and 88 women) referred to evaluation of spine surgery because of radicular symptoms in the Departments of Orthopedic Surgery and Neurosurgery at the Oulu University Hospital between June 2007 and January 2011. A neurosurgeon or orthopedic surgeon investigated the patients and a lumbar magnetic resonance imaging (MRI) was performed to all. Altogether $88 \%(\mathrm{n}=176)$ of patients had MRI-verified disc herniation (HNP)-induced nerve root impingement and major part of these patients were operated consequently. Twelve percent $(n=25)$ of patients did not have HNP-induced sciatica; about one half of them had radicular pain but in MRI only disc degeneration with or without bulge at the suspected level, while the rest had spondylolisthesis, nerve root canal narrowing or ligamentum flavum thickening. Patients with symptomatic spinal stenosis or spondylolisthesis on basis of the referral were excluded. The control subjects ( $\mathrm{n}=138$, age $42 \pm 11$ years, 39 men and 99 women) were healthy volunteers without low back pain, back disease, or back surgery and they were recruited to the study by the local occupational health care center (ODL Health, Oulu, Finland). All the subjects were asked not to eat or to drink coffee for $3 \mathrm{~h}$ before the tests. In addition, strenuous physical activity and alcohol consumption were prohibited on the day of the test and the preceding day. MRI of the lumbar spine was performed for both groups with 1.5-T equipment (Signa, General Electric, Milwaukee, WI, and Magnetom Avanto, Siemens, Erlangen, Germany). A routine spine MRI protocol was used, including sagittal T1- and T2-weighted images of the entire lumbar spine. The subjects' place of residence was mainly the city of Oulu (population ca. 140,000) or the neighboring municipalities, but the enrollment area was comprised of the two northernmost provinces of Finland (Oulu and Lapland). All the subjects filled out a baseline questionnaire. Potential confounders or modifying factors including comorbidities, leisure-time physical activity (LTPA), and smoking status were collected with the questionnaire. The demographics and clinical characteristics of the study cohort are presented in Table 1. The Ethical Committee of the Northern Ostrobothnia Hospital District, Oulu, Finland, approved the protocol, and all the patients gave informed consent.

Table 1 Demographic and clinical characteristics in patients referred to spine surgery and controls

\begin{tabular}{|c|c|c|c|}
\hline Variable & Patients $(n=201)$ & Controls $(n=138)$ & P-value \\
\hline Men/Women & $113(56) / 88(44)$ & $39(28) / 99(73)$ & $<0.0001$ \\
\hline Age, yr & $42 \pm 11$ & $42 \pm 11$ & 0.929 \\
\hline $\mathrm{BMl}, \mathrm{kg} / \mathrm{m}^{2}$ & $26.4 \pm 4.4$ & $23.9 \pm 3.6$ & $<0.0001$ \\
\hline Smoking & $124(62)$ & $49(36)$ & $<0.0001$ \\
\hline \multicolumn{4}{|l|}{ LTPA } \\
\hline High & $52(28)$ & $55(40)$ & 0.014 \\
\hline Moderate & $111(59)$ & $67(49)$ & 0.091 \\
\hline Low & $26(13)$ & $14(11)$ & 0.349 \\
\hline Oswestry, \% & $37 \pm 14$ & $2 \pm 3$ & $<0.0001$ \\
\hline VAS, cm & $5.8 \pm 2.5$ & $0.6 \pm 1.0$ & $<0.0001$ \\
\hline Pain Duration, days & $193 \pm 119$ & $7 \pm 16$ & $<0.0001$ \\
\hline Hypertension & $20(10)$ & $7(5)$ & 0.075 \\
\hline Diabetes & $3(1)$ & $1(<1)$ & 0.462 \\
\hline$C A D$ & $3(1)$ & $2(1)$ & 0.671 \\
\hline Hypercholesterolemia & $6(3)$ & $3(2)$ & 0.464 \\
\hline Asthma & $7(3)$ & $7(5)$ & 0.324 \\
\hline Arthritis or Fibromyalgia & $4(2)$ & $1(<1)$ & 0.329 \\
\hline
\end{tabular}

Values are means \pm SD or number of subjects (\%).

$\mathrm{BMI}=$ body mass index, LTPA = leisure-time physical activity, Oswestry = low back pain disability index, VAS = Visual Analog Scale, CAD = coronary artery disease. 


\section{Assessment of pain and disability}

All the subjects reported the duration of LBP during the past year. The diagnoses for surgical referral were obtained from hospital records. Back-related disability was assessed using the Oswestry Disability Index [17]. A 10-cm Visual Analog Scale (VAS) was used to define the intensity of LBP [18], with '0' representing a lack of pain and ' 10 ' the worst imaginable LBP.

\section{Assessment of heart rate variability}

A heart rate (HR) monitor (Polar RS800, Polar Electro Oy, Kempele, Finland) was used to record R-R intervals with a timing accuracy of 1 ms during 5 minutes ( $\mathrm{min}$ ) in a supine position followed by 5 min of standing in a quiet room after $5 \mathrm{~min}$ of rest in a supine position. The recordings were saved on a computer for further analysis of HRV with HEARTS software (Heart Signal Co, Kempele, Finland). To exclude all undesirable beats, which accounted for $<1 \%$ of the data for each subject, the R-R intervals were edited by visual inspection. HRV indices were calculated separately for the 5-min supine and standing conditions.

Mean HR and the standard deviation of normal-tonormal R-R intervals (SDNN) were used as time-domain measures of HRV. An autoregressive model (model 18) was used to estimate the power spectrum densities of R$\mathrm{R}$ interval variability. Vagally mediated HF power $(0.15$ to $0.4 \mathrm{~Hz}$ ), both sympathetically and vagally mediated low frequency power (LF, 0.04 to $0.15 \mathrm{~Hz}$ ), and the lowto-high-frequency ratio (LF/HF ratio) as an index of sympathovagal balance were calculated [19]. A Poincaré plot was analyzed quantitatively using two-dimensional vector analysis. Briefly, SD1 measures the magnitude of vagally mediated beat-to-beat variability in the data. Details of the two-dimensional vector analyses have been described previously elsewhere and were used accordingly in the present study [20]. As non-linear indices of HRV we analyzed the short-term $\left(\alpha_{1}\right)$ fractal scaling exponent. The fractal analysis method differs from traditional measures of HRV because it does not measure the magnitude of variability, but rather the qualitative characteristics and correlation features of HR behavior. Briefly, in the fractal analysis, the root-mean-square fluctuations of integrated and detrended data are measured in observation windows of different sizes and then plotted against the size of the window on a $\log$-log scale. In this study, short-term (from 4 to 11 beats) $\alpha_{1}$ was calculated on the basis of previous experiments [21,22]. Details of the fractal scaling method have been described previously elsewhere [23].

\section{Statistical analyses}

The normal Gaussian distribution of the data was verified with the Kolmogorov-Smirnov goodness-of-fit test.
Because the spectral components of HRV had a nonGaussian distribution, a natural logarithmic transformation was performed on both. Analysis of variance (ANOVA) was used to compare if the demographics and clinical characteristics and the mean values of HRV differed between the patients and the controls. A chisquare test was also used to compare categorical parameters. Analysis of covariance (ANCOVA) with 95\% confidence intervals $(\mathrm{CI})$ was performed to compare the differences in HRV between the groups, with gender, body mass index (BMI), smoking status, and high LTPA used as covariates. Pearson correlation analysis and ANOVA were applied to study the associations between HRV and demographics, disability, and pain-related parameters among the patients. Since underlying comorbidities may be associated with altered HRV, we excluded patients with hypertension, diabetes, coronary artery disease, hypercholesterolemia, asthma and arthritis or fibromyalgia from both patient and control populations and reperformed the statistical analyses. Furthermore, we performed separately a sub-analysis by comparing patients with and without HNP-induced sciatica. The SPSS statistical software package (IBM SPSS Statistics 20 for Windows, SPSS Inc., USA) was used for the analyses. A P-value $<0.05$ was considered statistically significant.

\section{Results}

\section{Study population characteristics}

The patients and controls did not differ with respect to age, co-morbidities, or moderate or low LTPA (Table 1). However, BMI was $10 \%$ higher in the patients than in the controls $(\mathrm{p}<0.0001)$ and there were more smokers among the patients than among the controls $(62 \%$ vs. $36 \%$, respectively, $\mathrm{p}<0.0001)$. There were more men (56\%) in the patient group compared with the controls $(28 \%)$ ( $\mathrm{p}<0.0001)$. High LTPA was reported among 40\% of the control subjects, whereas $28 \%$ of the patients showed high LTPA $(\mathrm{p}<0.014)$.

\section{Heart rate variability}

The differences in HRV between the patients referred to spine surgery and the controls are presented in Table 2. HR was 4 beats higher among the patients in a supine position compared with the controls $(\mathrm{p}=0.002)$ and 7 beats higher in a standing position $(\mathrm{p}<0.0001$, Figure 1A). The difference in all HRV parameters between the patients and the controls $(p=0.002$ to $\mathrm{p}<0.0001$ ) in a standing position was statistically significant, e.g. vagally mediated HF power (Figure 1B) was $13 \%$ lower and the LF/HF ratio, as an index of sympathovagal balance, was $36 \%$ higher in the patients than in the controls. After adjustment for gender, BMI, smoking, and high LTPA, the statistical difference between the groups in a standing position remained 
Table 2 Heart rate variability indices in patients referred to spine surgery and controls during $\mathbf{5}$ minutes of supine and standing conditions

\begin{tabular}{|c|c|c|c|c|c|c|c|c|}
\hline & \multicolumn{4}{|l|}{ Supine } & \multicolumn{4}{|l|}{ Standing } \\
\hline & Patients & Controls & $\begin{array}{l}\text { Mean difference } \\
\text { (95\% Cl for difference) }\end{array}$ & $\overline{\text { Adj. P-value }}$ & Patients & Controls & $\begin{array}{l}\text { Mean difference } \\
\text { (95\% Cl for difference) }\end{array}$ & Adj. P-value \\
\hline $\mathrm{HR}$, bpm & $69 \pm 13$ & $65 \pm 10$ & $3(0-6)$ & 0.051 & $85 \pm 14$ & $78 \pm 13$ & $7(3-10)$ & $<0.0001$ \\
\hline SDNN, ms & $45 \pm 28$ & $48 \pm 24$ & $2(-5-9)$ & 0.598 & $51 \pm 22$ & $61 \pm 27$ & $-6(-12-0)$ & 0.065 \\
\hline $\begin{array}{l}\text { HF power, } \\
\ln \mathrm{ms}^{2}\end{array}$ & $5.6 \pm 1.4$ & $6.0 \pm 1.3$ & $-0.2(-0.5-0.2)$ & 0.356 & $4.5 \pm 1.2$ & $5.1 \pm 1.2$ & $-0.4(-0.7--0.1)$ & 0.011 \\
\hline $\begin{array}{l}\text { LF power, } \\
\text { In } \mathrm{ms}^{2}\end{array}$ & $6.0 \pm 1.1$ & $6.1 \pm 1.0$ & $-0.1(-0.4-0.2)$ & 0.404 & $6.3 \pm 1.1$ & $6.6 \pm 0.9$ & $-0.2(-0.5-0.1)$ & 0.074 \\
\hline $\begin{array}{l}\text { LF-to-HF } \\
\text { ratio }\end{array}$ & $2.0 \pm 1.7$ & $1.9 \pm 2.3$ & $-0.3(-0.8-0.2)$ & 0.205 & $7.9 \pm 6.4$ & $5.8 \pm 5.2$ & $1.0(-0.4-2.5)$ & 0.159 \\
\hline $\mathrm{SD} 1, \mathrm{~ms}$ & $23 \pm 15$ & $28 \pm 21$ & $-2(-6-3)$ & 0.443 & $13 \pm 7$ & $18 \pm 12$ & $-4(-6--1)$ & 0.002 \\
\hline$a_{1}$ & $1.09 \pm 0.23$ & $1.01 \pm 0.29$ & $0.03(-0.03-0.10)$ & 0.304 & $1.58 \pm 0.22$ & $1.49 \pm 0.24$ & $0.73(0.02-0.13)$ & 0.012 \\
\hline
\end{tabular}

Values are means \pm SD. Mean differences and $95 \%$ confidence intervals $(\mathrm{Cl})$ for differences between groups are given after adjustment for gender, $\mathrm{BMI}$, smoking status and high LTPA.

$\mathrm{HR}=$ heart rate, $\mathrm{SDNN}=$ standard deviation of all $\mathrm{R}-\mathrm{R}$ intervals, $\mathrm{HF}=$ high frequency, $\mathrm{LF}=$ low frequency, $\mathrm{SD} 1=$ beat to beat $\mathrm{R}-\mathrm{R}$ interval fluctuation, $\mathrm{a}_{1}=$ short-term fractal scaling exponent.

evident for HR, HF power, SD1, and $\alpha_{1}$ (Table 2). When underlying co-morbidities were excluded from the analyses, the results remained the same; e.g. HF power, SD1, and $\alpha_{1}$ in standing position differed significantly between the groups after adjustments $(\mathrm{p}=0.048, \mathrm{p}=0.012$ and $\mathrm{p}=0.010$, respectively). Furthermore, when comparing patients with and without HNP-induced sciatica, patients without HNP-induced sciatica were older $(50 \pm 7$ vs. $40 \pm$ $11, \mathrm{p}<0.0001)$ and the duration of pain was longer $(266 \pm$ 139 vs. $181 \pm 107$ days, $\mathrm{p}=0.002$ ) compared to patients with HNP-induced sciatica. The two patient groups differed also in HRV; e.g. HF power in standing position was $5.63 \pm 1.36$ in $\mathrm{HNP}$-induced sciatica patients and $4.99 \pm 1.36(\mathrm{p}=0.002)$ in patients without HNPinduced sciatica. However, after adjustment with age and pain duration the difference disappeared in all
HRV parameters (e.g. in HF power in standing position $\mathrm{p}=0.211)$.

\section{Relationships between heart rate variability and other variables in the patients}

The Oswestry and VAS scores did not correlate with HRV, e.g. the correlation values between HF power and the Oswestry score and HF power and the VAS score in a standing position were -0.036 and -0.094 , respectively. Pain duration was inversely associated with standing HF power $(\mathrm{r}=-.232, \mathrm{p}=0.003)$ but not with $\mathrm{HR}, \mathrm{SD} 1$, or $\alpha_{1}$ $(\mathrm{r}=-0.032,-0.111,0.001$, respectively). Similar results were found when underlying co-morbidities were taken into consideration; e.g. pain duration correlated with standing HF power $(\mathrm{r}=-.217, \mathrm{p}=0.009)$. Age and BMI were negatively associated with standing HF power
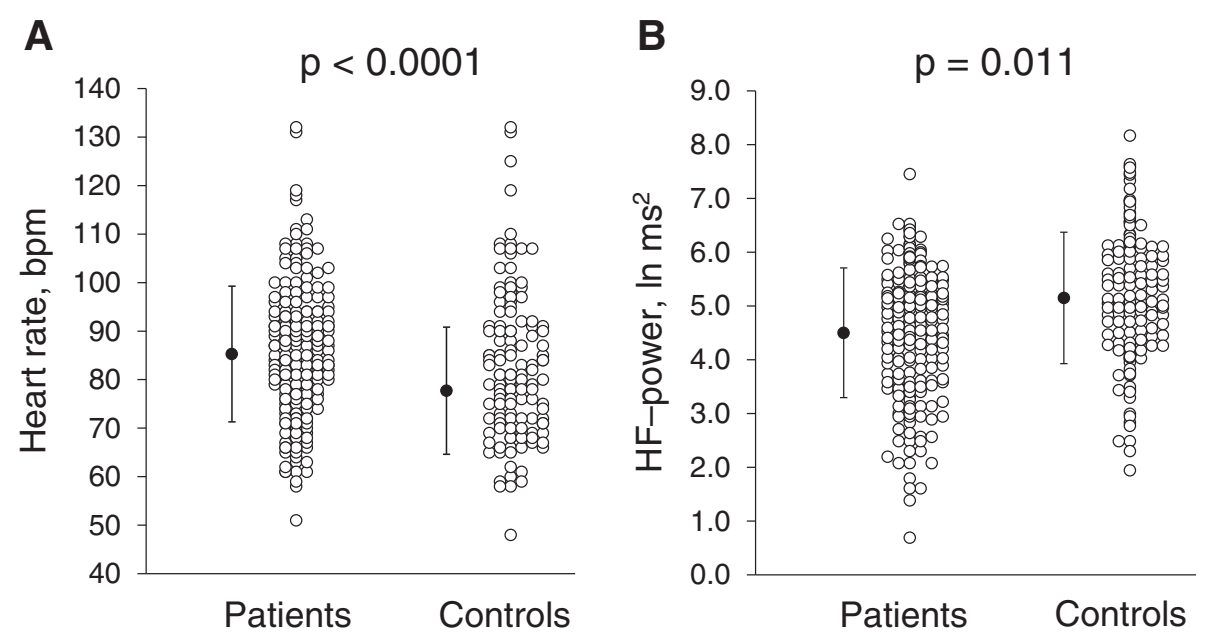

Figure 1 Mean heart rate (A) and vagally mediated HF power (B) in a standing position for 5 minutes for patients and controls. 
$(\mathrm{r}=-0.408, \mathrm{p}<0.0001$ and $\mathrm{r}=-0.169, \mathrm{p}=0.017$, respectively). In a subgroup analysis according to smoking status or LTPA among the patients, standing HR, HF power, SD1, or $\alpha_{1}$ did not differ from each other ( $\mathrm{p}=\mathrm{ns}$ for all).

\section{Discussion}

We have shown that autonomic nervous system function assessed with the HRV method differs between sciatica patients referred to spine surgery and healthy controls. Vagally mediated HF power and SD1 were significantly lower in the patients than in the healthy controls, whereas the short-term fractal scaling exponent $\alpha_{1}$, indicating sympathovagal balance, was higher in the patients than in the controls after adjusting for potential confounders. Impaired functional capacity caused by pain or pain intensity was not associated with HRV among the patients, but HF power correlated negatively with LBP duration. Since altered HRV has been shown to have a prognostic value in both healthy subjects and various patient groups, the present results may provide important clues for understanding and considering the contribution of autonomic nervous system function in sciatica patients referred to spine surgery.

\section{Heart rate variability among low back patients}

It is well documented that gender, BMI, smoking, and physical activity affects HRV [24-27]. Although there were more smokers, higher BMI, less high LTPA, and more men in the patient group than in the controls, HRV still differed between the groups when these potential confounders were accounted for. Additionally, the association remained evident after accounting for underlying co-morbidities and in separate sub-analysis where the patients with and without HNP-induced sciatica were compared. Taken together, obvious differences between the patient and control groups were observed in pain intensity (VAS score) and perceived disability (Oswestry), emphasizing the independent role of pain in HRV.

In a previous study, higher HF power was observed among patients with chronic LBP whose Oswestry score was less than $20 \%$ compared with patients whose score was $20-40 \%$, but HF power did not differ between patients whose VAS score was $0-5$ vs. 6-10 [10]. In the present study, we could not find an association between HF power and Oswestry or VAS score, whereas HF power correlated with pain duration, i.e. decreased vagal outflow was related to prolonged pain. Since in our study attenuated vagal activity during orthostatic stress was not linked to pain intensity or impaired functional capacity, it might be aggravated by the chronicity of the disease measured by the duration of pain. This finding may suggest that the dysfunction of the autonomic nervous system is a feature of the current pain condition and not related to pain severity per se.

\section{Potential mechanisms for decreased heart rate variability among the patients}

It has been evidenced for a long time that various states of chronic pain, including LBP, are associated with signs of altered autonomic nervous system function, but the physiological mechanisms remain unclear. A recent study showed that HRV was reduced in complex regional pain syndrome (CRPS) patients compared with controls [8]. During orthostatic stress, patients with CRPS were not able to preserve cardiac output and they had an exaggerated increase in total peripheral resistance. Interestingly, the hemodynamic changes correlated with pain duration but not with pain intensity, e.g. a tiltinduced increase in total peripheral resistance correlated positively with pain duration [8]. Furthermore, in patients with chronic widespread pain (CWP), lower HRV is associated with higher pain intensity [28]. Interestingly, a recent study by Kang et al. showed that different disease profiles can be determined by clustering distinct features in patients with chronic neck pain [29]. According to this study, a high degree of disability associated with low HRV, high pain intensity, older age, poor sleep quality and high psychological distress. These findings may suggest a general autonomic imbalance in both CRPS, CWP and chronic neck pain patients, which may also be the status of our sciatica patients referred to spine surgery.

Recent studies have shown that the production of proinflammatory markers within the nucleus pulposus may be an important mediator in discogenic pain. Elevated levels of serum interleukin-6 (IL-6) and tumor necrosis factor- $\alpha$ (TNF- $\alpha$ ) in patients with a herniated lumbar intervertebral disc [30] or elevated IL-6 in patients with lumbar radiculopathy [31], compared with healthy subjects, have been observed. Interestingly, autonomic dysregulation, expressed as depressed HRV, has been linked to increased IL- 6 in healthy individuals as well as in those with a cardiovascular disease [32,33]. Therefore, it could be hypothesized that autonomic dysfunction observed in the sciatica patients in the present study may be related to increased cytokine production by the immune system. However, this should be confirmed in future studies.

\section{Methodological considerations}

In the present study, we assessed HRV in a supine position and also during orthostatic stress in a standing position. It is well documented that HRV is susceptible to saturation when measured at a low $\operatorname{HR}[34,35]$, which is usually the case in a supine position, and it may therefore be unable to detect changes in cardiac vagal 
outflow [36]. Additionally, vagal and sympathetic regulation operates in a reciprocal fashion during a sympathetic orthostatic stimulus $[37,38]$. Therefore, a standing position might be a more favorable condition than supine for measuring autonomic function, since it could detect attenuated vagal outflow related to increased sympathetic activity, as well. Indeed, in the present study we found a clearer difference in HRV indices measured in a standing position then in a supine position.

It is also noteworthy that we measured the fractal scaling exponent $\alpha_{1}$ as an index of sympathovagal balance, because this index has been shown to provide information on autonomic interactions during various interventions and is less sensitive than spectral measures to trends and non-stationarities in HR behavior [23,39-41]. In the present study, the LF/HF ratio was higher in the patients than in the controls in a standing position, but after adjusting for sex, smoking, BMI, and LTPA, the difference between the groups disappeared $(p=n s)$. However, the difference in $\alpha_{1}$ between the groups remained evident ( $p=0.012)$ after adjusting for the abovementioned covariates, indicating the role of both reduced vagal and increased sympathetic outflow among the patients.

\section{Limitations}

Uncontrolled factors such as the night-time sleep or other psychological and physiological stressors may contribute to daily HRV. Our subjects were voluntary consecutive patients or healthy controls scheduled to daily hospital routine, and we were not able to control the above-mentioned factors. In addition, we could not control the use of pain medications, which may have an effect on HRV. Even though we may be able to evaluate pain medications according to hospital databases, we do not know the quality and amount of over-the-counter pain medication. However, we believe that most of the patients used pain medication at least to some extent while control population did not. This should "dilute" the results as pain medication is supposed to alleviate pain symptoms. Unfortunately, we do not have knowledge about use of physical therapy but we do not regard it an important confounder as it (if it works) has similar effect as pain medication. However, we analysed the data when underlying co-morbidities were excluded and the results were the same. Obviously, subjects with comorbidities use more likely medication than subjects without co-morbidities. Therefore, we feel that, at least to some extent, medication is taken into consideration.

\section{Implications}

Reduced HRV has been shown to be associated with the occurrence of various clinical events. Our results indicate that sciatica patients referred to spine surgery had autonomic dysregulation with decreased vagal activity and an increased sympathovagal balance compared with age-matched healthy controls. Therefore, it seems important to consider monitoring of the autonomic nervous system in addition to pain management in sciatica patients. We did not observe a significant relationship between impaired functional capacity caused by pain or pain intensity and HRV. In this respect, it can be suggested that HRV may reflect an independent intrinsic regulatory system of the autonomic nervous system that may be affected by the severity of the sciatic disease.

\section{Conclusions}

These data suggest that sciatica patients referred to spine surgery have decreased HRV when compared with healthy subjects, and this may reflect a predisposition to impaired autonomic cardiovascular regulation.

\section{Abbreviations}

BMI: Body mass index; CAD: Coronary artery disease; CRPS: Complex regional pain syndrome; CWP: Chronic widespread pain; HF: High frequency;

HNP: Disc herniation induced nerve root impingement; HR: Heart rate HRV: Heart rate variability; IL-6: Interleukin-6; LBP: Low back pain; LF: Low frequency; LF/HF: Low-to-high-frequency ratio; LTPA: Leisure-time physica activity; MRI: Magnetic resonance imaging; Oswestry: Low back pain disability index; SDNN: Standard deviation of all R-R intervals; SD1: Beat to beat R-R interval fluctuation; TNF-a: Tumor necrosis factor-a; VAS: Visual analog scale; $a_{1}=$ : Short-term fractal scaling exponent.

\section{Competing interests}

The authors declare that they have no financial or non-financial competing interests.

\section{Authors' contributions}

$J \mathrm{~K}, \mathrm{AJH}$ and JS conceived the study and participated in its design. JK, AJH and JS had also full access to all of the data in the study and take responsibility for the integrity of the data and the accuracy of the data analyses and final version of the manuscript. JK, JP and EK coordinated and carried out the clinical data of the patients, participated the data analysis and drafted the manuscript. AMK and MPT participated in the analysis and interpretation of data and helped to draft the manuscript. AJH performed the statistical analysis together with AMK and MPT. All authors read and approved the final manuscript.

\section{Acknowledgments}

The authors express their gratitude to Ms Anna Laulumaa and Ms Jenny Siltala for the help with collection of data.

\section{Author details}

'Department of Exercise and Medical Physiology, Verve Research, Kasarmintie 13, P.O. Box 404, 90101 Oulu, Finland. ${ }^{2}$ Department of Physical and Rehabilitation Medicine, Institute of Clinical Medicine, University of Oulu, Oulu, Finland. ${ }^{3}$ Finnish Institute of Occupational Health, Work and Health Ability, and Disability Prevention Centre, Oulu, Finland.

Received: 8 October 2012 Accepted: 23 April 2013

Published: 26 April 2013

\section{References}

1. Breivik H, Collett B, Ventafridda V, Cohen R, Gallacher D: Survey of chronic pain in Europe: prevalence, impact on daily life, and treatment. Eur J Pain 2006, 10:287-333.

2. Konstantinou K, Dunn KM: Sciatica: review of epidemiological studies and prevalence estimates. Spine (Phila Pa 1976) 2008, 33:2464-2472. 
3. Kaaria S, Kaila-Kangas L, Kirjonen J, Riihimaki H, Luukkonen R, Leino-Arjas $P$ : Low back pain, work absenteeism, chronic back disorders, and clinical findings in the low back as predictors of hospitalization due to low back disorders: a 28-year follow-up of industrial employees. Spine (Phila Pa 1976) 2005, 30:1211-1218.

4. Riihimaki H, Tola S, Videman T, Hanninen K: Low-back pain and occupation. A cross-sectional questionnaire study of men in machine operating, dynamic physical work, and sedentary work. Spine (Phila PC 1976) 1989, 14:204-209.

5. Abasolo L, Carmona L, Lajas C, Candelas G, Blanco M, Loza E, Hernandez-Garcia C, Jover JA: Prognostic factors in short-term disability due to musculoskeletal disorders. Arthritis Rheum 2008, 59:489-496.

6. Cohen H, Neumann L, Shore M, Amir M, Cassuto Y, Buskila D: Autonomic dysfunction in patients with fibromyalgia: application of power spectral analysis of heart rate variability. Semin Arthritis Rheum 2000, 29:217-227.

7. Cohen H, Neumann L, Alhosshle A, Kotler M, Abu-Shakra M, Buskila D: Abnormal sympathovagal balance in men with fibromyalgia. J Rheumatol 2001, 28:581-589.

8. Terkelsen AJ, Molgaard H, Hansen J, Finnerup NB, Kroner K, Jensen TS: Heart rate variability in complex regional pain syndrome during rest and mental and orthostatic stress. Anesthesiology 2012, 116:133-146.

9. Hallman DM, Lindberg LG, Arnetz BB, Lyskov E: Effects of static contraction and cold stimulation on cardiovascular autonomic indices, trapezius blood flow and muscle activity in chronic neck-shoulder pain. Eur J Appl Physiol 2011, 111:1725-1735.

10. Gockel M, Lindholm H, Niemisto L, Hurri H: Perceived disability but not pain is connected with autonomic nervous function among patients with chronic low back pain. J Rehabil Med 2008, 40:355-358.

11. Huikuri HV, Castellanos A, Myerburg RJ: Sudden death due to cardiac arrhythmias. N Engl J Med 2001, 345:1473-1482.

12. Bauer A, Kantelhardt JW, Barthel P, Schneider R, Makikallio T, Ulm K, Hnatkova K, Schomig A, Huikuri H, Bunde A, et al: Deceleration capacity of heart rate as a predictor of mortality after myocardial infarction: cohort study. Lancet 2006, 367:1674-1681.

13. Huikuri HV, Makikallio TH, Airaksinen KE, Seppanen T, Puukka P, Raiha IJ, Sourander LB: Power-law relationship of heart rate variability as a predictor of mortality in the elderly. Circulation 1998, 97:2031-2036.

14. Kleiger RE, Miller JP, Bigger JT Jr, Moss AJ: Decreased heart rate variability and its association with increased mortality after acute myocardial infarction. Am J Cardiol 1987, 59:256-262.

15. Bigger JT Jr, Fleiss JL, Steinman RC, Rolnitzky LM, Kleiger RE, Rottman JN: Frequency domain measures of heart period variability and mortality after myocardial infarction. Circulation 1992, 85:164-171.

16. Kiviniemi AM, Tulppo MP, Wichterle D, Hautala AJ, Tiinanen S, Seppanen T, Makikallio TH, Huikuri HV: Novel spectral indexes of heart rate variability as predictors of sudden and non-sudden cardiac death after an acute myocardial infarction. Ann Med 2007, 39:54-62.

17. Fairbank JC, Pynsent PB: The Oswestry Disability Index. Spine (Phila Pa 1976) 2000, 25:2940-2952. discussion 2952.

18. Sriwatanakul K, Kelvie W, Lasagna L, Calimlim JF, Weis OF, Mehta G: Studies with different types of visual analog scales for measurement of pain. Clin Pharmacol Ther 1983, 34:234-239.

19. TaskForce: Heart rate variability: standards of measurement, physiological interpretation and clinical use. Task Force of the European Society of Cardiology and the North American Society of Pacing and Electrophysiology. Circulation 1996, 93:1043-1065.

20. Tulppo MP, Makikallio TH, Takala TE, Seppanen T, Huikuri HV: Quantitative beat-to-beat analysis of heart rate dynamics during exercise. Am J Physiol 1996, 271:H244-H252.

21. Huikuri HV, Makikallio TH, Peng CK, Goldberger AL, Hintze U, Moller M: Fractal correlation properties of R-R interval dynamics and mortality in patients with depressed left ventricular function after an acute myocardial infarction. Circulation 2000, 101:47-53.

22. Makikallio TH, Huikuri HV, Makikallio A, Sourander LB, Mitrani RD, Castellanos A, Myerburg RJ: Prediction of sudden cardiac death by fractal analysis of heart rate variability in elderly subjects. J Am Coll Cardiol 2001, 37:1395-1402

23. Peng CK, Havlin S, Stanley HE, Goldberger AL: Quantification of scaling exponents and crossover phenomena in nonstationary heartbeat time series. Chaos 1995, 5:82-87.
24. Huikuri HV, Pikkujämsä SM, Airaksinen KE, Ikäheimo MJ, Rantala AO, Kauma H, Lilja M, Kesäniemi YA: Sex-related differences in autonomic modulation of heart rate in middle-aged subjects. Circulation 1996, 94:122-125.

25. Hautala AJ, Karjalainen J, Kiviniemi AM, Kinnunen H, Makikallio TH, Huikuri HV, Tulppo MP: Physical activity and heart rate variability measured simultaneously during waking hours. Am J Physiol Heart Circ Physiol 2010 298:H874-H880

26. Hayano J, Yamada M, Sakakibara Y, Fujinami T, Yokoyama K, Watanabe Y, Takata K: Short- and long-term effects of cigarette smoking on heart rate variability. Am J Cardiol 1990, 65:84-88.

27. Fagard RH, Pardaens K, Staessen JA: Influence of demographic, anthropometric and lifestyle characteristics on heart rate and its variability in the population. J Hypertens 1999, 17:1589-1599.

28. Barakat A, Vogelzangs N, Licht CM, Geenen R, Macfarlane GJ, de Geus E, Smit JH, Penninx BW, Dekker J: Dysregulation of the autonomic nervous system is associated with pain intensity, not with the presence of chronic widespread pain. Arthritis Care Res (Hoboken) 2012, 64:1209-1216.

29. Kang JH, Chen HS, Chen SC, Jaw FS: Disability in patients with chronic neck pain: heart rate variability analysis and cluster analysis. Clin J Pain 2012, 28:797-803.

30. Kraychete DC, Sakata RK, Issy AM, Bacellar O, Santos-Jesus R, Carvalho EM: Serum cytokine levels in patients with chronic low back pain due to herniated disc: analytical cross-sectional study. Sao Paulo Med J 2010, 128:259-262.

31. Nagashima H, Morio $Y$, Yamane $K$, Nanjo $Y$, Teshima R: Tumor necrosis factor-alpha, interleukin-1beta, and interleukin-6 in the cerebrospinal fluid of patients with cervical myelopathy and lumbar radiculopathy. Eur Spine J 2009, 18:1946-1950.

32. Lampert R, Bremner JD, Su S, Miller A, Lee F, Cheema F, Goldberg J, Vaccarino V: Decreased heart rate variability is associated with higher levels of inflammation in middle-aged men. Am Heart J 2008, 156:759 e751-757.

33. Haensel A, Mills PJ, Nelesen RA, Ziegler MG, Dimsdale JE: The relationship between heart rate variability and inflammatory markers in cardiovascular diseases. Psychoneuroendocrinology 2008, 33:1305-1312.

34. Kiviniemi AM, Hautala AJ, Seppänen T, Mäkikallio TH, Huikuri HV, Tulppo MP. Saturation of high-frequency oscillations of R-R intervals in healthy subjects and patients after acute myocardial infarction during ambulatory conditions. Am J Physiol Heart Circ Physiol 2004, 287:H1921-1927.

35. Goldberger JJ, Challapalli S, Tung R, Parker MA, Kadish AH: Relationship of heart rate variability to parasympathetic effect. Circulation 2001, 103:1977-1983.

36. Kiviniemi AM, Hautala AJ, Kinnunen H, Tulppo MP: Endurance training guided individually by daily heart rate variability measurements. Eur J Appl Physiol 2007, 101:743-751.

37. Malliani A, Pagani M, Lombardi F, Cerutti S: Cardiovascular neural regulation explored in the frequency domain. Circulation 1991, 84:482-492.

38. Montano N, Ruscone TG, Porta A, Lombardi F, Pagani M, Malliani A: Power spectrum analysis of heart rate variability to assess the changes in sympathovagal balance during graded orthostatic tilt. Circulation 1994, 90:1826-1831.

39. Tulppo MP, Hughson RL, Mäkikallio TH, Airaksinen KE, Seppänen T, Huikuri HV: Effects of exercise and passive head-up tilt on fractal and complexity properties of heart rate dynamics. Am J Physiol Heart Circ Physiol 2001, 280:H1081-1087.

40. Tulppo MP, Kiviniemi AM, Hautala AJ, Kallio M, Seppänen T, Mäkikallio TH, Huikuri HV: Physiological background of the loss of fractal heart rate dynamics. Circulation 2005, 112:314-319.

41. Hautala AJ, Makikallio TH, Seppanen T, Huikuri HV, Tulppo MP: Short-term correlation properties of R-R interval dynamics at different exercise intensity levels. Clin Physiol Funct Imaging 2003, 23:215-223.

doi:10.1186/1471-2474-14-149

Cite this article as: Södervall et al: Heart rate variability in sciatica patients referred to spine surgery: a case control study. BMC Musculoskeletal Disorders 2013 14:149. 